\title{
Generalized Joint Hypermobility Is Associated With Decreased Hip Labrum Width: A Magnetic Resonance Imaging-Based Study
}

\author{
Jonathan D. Haskel, M.D., Daniel J. Kaplan, M.D., Noah Kirschner, M.D., \\ Jordan W. Fried, B.M., Mohammad Samim, M.D., Christopher Burke, M.B., Ch.B., and \\ Thomas Youm, M.D.
}

\begin{abstract}
Purpose: To explore the relationship between generalized joint hypermobility and hip labrum width. Methods: A retrospective review was performed of a single-surgeon database containing patients who underwent hip arthroscopy between 2014 and 2017. Patients were assessed for generalized laxity via Beighton Test Scoring (BTS), which tests for hyperextension of the fifth metacarpophalangeal joint, thumb apposition, elbow hyperextension, knee hyperextension, and trunk flexion on a 9-point scale. Patients were stratified into a "high BTS cohort" with a BTS $\geq 4$, and a control cohort with BTS $<4$. Magnetic resonance imaging measurements of labral width for each patient were conducted by 2 blinded, musculoskeletal fellowship-trained radiologists at standardized "clockface" locations using a previously validated technique. Statistical analyses used to determine associations between BTS and labral width included Mann-Whitney $U$ and Fisher exact testing as well as linear regression. Results: Thirty-four patients met inclusion criteria (17 cases, 17 controls). Both groups were composed exclusively of female patients. There was no significant difference between cases or controls in terms of age $(33.3 \pm 10.4$ years vs $35.2 \pm 8.3$ years, $P=.57)$ or body mass index $(26.1 \pm 9.3$ vs $23.6 \pm 3.4, P=.36)$. The high Beighton score cohort had significantly thinner labrae at the indirect rectus $(5.35 \pm 1.2 \mathrm{~mm}$ vs $7.1 \pm 1.1 \mathrm{~mm}, P<$ $.001)$ and anterosuperior position $(5.53 \pm 1.4 \mathrm{~mm}$ vs $7.27 \pm 1.6 \mathrm{~mm}, P=.003)$. There was no statistical difference between the high Beighton score cohort and controls at the psoas $U$ position $(6.47 \pm 1.6 \mathrm{~mm}$ vs $7.43 \pm 1.7 \mathrm{~mm}, P=.112)$. Linear regression analysis demonstrated Beighton score was significantly negatively associated with labrum width at the indirect rectus position $\left(\mathrm{R}^{2}=0.33, P<.001\right)$ and the anterosuperior position $\left(\mathrm{R}^{2}=0.25, P=.004\right)$. Conclusions: Patients with a BTS $\geq 4$ were found to have significantly thinner labra than those with a BTS of $<4$. Level of Evidence: III, retrospective comparative trial.
\end{abstract}

$\mathbf{T}$ he intact labrum increases the acetabular articular surface area by a mean of $22 \%$ and the acetabular volume by a mean of $33 \% .^{1}$ These characteristics ultimately contribute to the seal effect conferred by the hip labrum, thus increasing stability within the hip

Departments of Orthopedic Surgery (J.D.H., D.J.K., N.K., J.W.F., T.Y.) and Radiology (M.S., C.B.), NYU Langone Health, New York, New York, U.S.A.

The authors report the following potential conflicts of interest or sources of funding: T.Y. reports other from Arthrex, outside the submitted work. Full ICMJE author disclosure forms are available for this article online, as supplementary material.

Received September 10, 2020; accepted January 24, 2021.

Address correspondence to Jonathan D. Haskel, M.D., 301 E 17th St., New York, NY 10003.E-mail: jonathan.haskel@nyumc.org

(C) 2021 THE AUTHORS. Published by Elsevier Inc. on behalf of the Arthroscopy Association of North America. This is an open access article under the CC BY-NC-ND license (http://creativecommons.org/licenses/by-nc-nd/4.0/). 2666-061X/201533

https://doi.org/10.1016/j.asmr.2021.01.017 joint. ${ }^{1}$ When the labrum is torn, there is an alteration in hip biomechanics such that there is an increase in anterior translation and external rotation of the femoral head within the acetabulum. ${ }^{2,3}$ In addition, there are increased contact stresses between the articular surfaces in the hip joint. ${ }^{1,4}$ Therefore, repairing the hip labrum is thought to improve patient outcomes by restoring native hip biomechanics and minimizing hip instability.

Generalized joint hypermobility (GJH) is a relatively common condition, with an overall prevalence in the healthy populations of $26.2 \% .^{5}$ Naal et al. ${ }^{6}$ have identified that the prevalence of $\mathrm{GJH}$ (as measured by Beighton test scoring) is greater in patients with femoroacetabular impingement (FAI) $(32.7 \%)$. Hip microinstability is extra-physiologic hip motion that causes pain and may be the result of bony deficiency and/or soft-tissue loss. ${ }^{7}$ Hip microinstability from GJH is believed to place increased force and tension on the hip capsule, which can predispose to labral injuries. ${ }^{8}$ As 
patients with laxity are reliant on soft-tissue structures for hip stability, it behooves hip arthroscopists to have a strong understanding of how the soft-tissue stabilizers within the hip joint work to augment stability.

Width has been identified as the most important component of the labrum with regards to conferring the suction seal effect. ${ }^{9,10}$ A recent study demonstrated that decreased labral width is associated with significantly worse functional outcomes following arthroscopic labral repair and treatment of FAI. ${ }^{11}$ While it has been established that GJH is associated with hip capsule thickness and microinstability, there is limited information on how GJH relates to labral width. Since patients with high Beighton scores are at increased risk for developing labral injuries, decreased labral width may explain why patients with GJH are more likely to incur hip labral injuries. A deeper appreciation of GJH could have implications on clinical outcomes following hip labral repair.

The purpose of this study was to explore the relationship between GJH and hip labrum width. The hypothesis was that smaller labral width would be associated with increased GJH.

\section{Methods}

This was a single-center, single-surgeon, retrospective study performed at a tertiary care center from 2014 to 2017. Before enrollment, the design and protocol were approved by the authors' institutional review board. All patients undergoing hip arthroscopy were included in an institutional database. Inclusion criteria for the current study consisted of age 18 to 65 years old, closed physes on radiograph, and hip arthroscopy based on diagnosis of FAI determined by clinical assessment and imaging studies (alpha-angle $>55^{\circ}$, lateral center edge angle $>40^{\circ}$, proximal acetabular retroversion. ${ }^{6}$ Exclusion criteria were defined as patients with previous ipsilateral hip surgery, history or radiographic evidence of hip dysplasia, Tönnis grade $>1$, ossified or calcified labra, inability to access magnetic resonance imaging (MRI) in PACS or lack of MRI, and/or lack of Beighton score. Hip dysplasia was defined as a lateral center-edge angle $<20^{\circ}$, Tönnis angle $>10^{\circ}$, Sharp's angle $>33^{\circ}$, vertical center anterior angle $<25^{\circ}$ (when false-profile view available), or femoral head extrusion index of $<25 \% .^{12}$ Demographic patient information including age, sex, laterality, and body mass index (BMI) were recorded. Patients with dysplasia were excluded, as it has been shown that they may have hypertrophic labra. ${ }^{13}$

\section{Diagnosis of GJH}

All patients underwent Beighton scoring upon initial presentation to the office. The technique for obtaining Beighton Test Scoring (BTS) was performed as described by Boyle et al. ${ }^{14}$ It involves 9 separate range of motion measurements as follows: trunk and hip flexion-a positive test defined as the ability to place the palms flat on the floor while keeping the knees extended ( 1 point); bilateral knee extension-a positive test defined as at least $10^{\circ}$ of hyperextension ( 1 point for each knee); bilateral elbow extension-a positive test defined as at least $10^{\circ}$ of hyperextension ( 1 point for each elbow); bilateral thumb to forearm apposition-a positive test defined as the ability to appose the thumb and volar aspect of the forearm ( 1 point for each upper extremity); bilateral little finger extension - a positive test defined as at least $90^{\circ}$ of hyperextension (1 point for each upper extremity). Patients with scores $\geq 4$ are considered to have GJH. ${ }^{15}$

\section{Diagnosis of FAl}

Clinical assessment suggestive of FAI included a reported history of groin or hip pain exacerbated by hip flexion or mechanical symptoms such as clicking or locking and physical exam signs including a positive flexion, adduction, internal rotation test or a positive flexion, abduction, external rotation test. Patients were assessed by the senior author, a sports medicine fellowship-trained orthopaedic surgeon with 15 years of experience. Other demographic information queried for the study included age, BMI, laterality, and presence of a connective tissue disorder. A patient was considered to have a connective tissue disorder if he/ she carried the diagnosis of Ehlers Danlos syndrome, Marfan syndrome, Raynaud disease, or systemic lupus erythematosus.

\section{Imaging}

MRIs included in this study were required to meet the following criteria; available in Picture Archiving and Communication System (PACS), performed on 1.5T MRI, 3T MRI, or 3T magnetic resonance angiography adequate quality as determined by reading radiologists (e.g., there was no artifact from patient movement, sequences were formatted in the correct planes by techs at time of imaging), and lack of labral ossification. Imaging from multiple types of MRI scanners were used as patients often elect to have the imaging study performed at independent locations with different machines. Based on a previous study, variation in magnet strength does not appear to pose an issue. ${ }^{16}$

\section{Group Formation}

Once the cohort of patients with high Beighton scores was selected, patients were matched to a control cohort with Beighton scores $<4$ in 1: 1 fashion by age, sex, and BMI. Controls were selected if they were within 2 years of the mean age, female sex, and within 3 points of the mean BMI. 

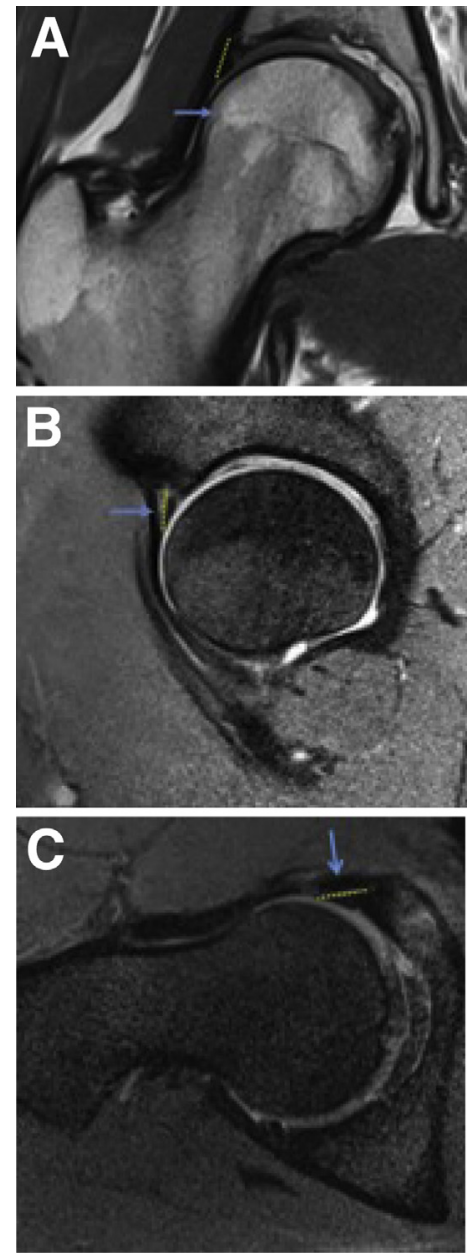

Fig 1. Magnetic resonance imaging (proton density sequence) demonstrating right hip labrum measurements at standardized positions. From left to right, indirect rectus insertion (1 1:30 o'clock) position measured in the (A) coronal plane without fat suppression, point midway between indirect rectus insertion and psoas $U$ position measured in the sagittal plane, (B) fat-suppressed, and (C) psoas U (3 o'clock) position measured in the axial plane, non-fat-suppressed.

\section{Radiologic Assessment}

\section{Imaging Techniques}

All MRI and magnetic resonance angiography studies were performed on either a 1.5T or 3T MR scanner (Magnetom Aera or Skyra; Siemens Medical Solutions, Erlangen, Germany) using an 18-channel body matrix coil. Imaging conducted at the study institution was automatically loaded into PACS. Imaging conducted outside of the study institution was uploaded to PACS via compact disc. A summary of the hip MRI protocol used can be seen in a previously published study.

\section{Imaging Evaluation}

Imaging evaluation was performed using a previously validated technique ${ }^{16}$. In brief, measurements were made independently by 2 musculoskeletal fellowship-trained radiologists, with 7 and 9 years of experience, who were blinded to clinical information and arthroscopic measurements. Reads were done on a PACS workstation (IntelliSpace PACS Enterprise; Philips North America, Andover, MA). All measurements were done using the measurement ruler tool of our institution's PACS workstation (IntelliSpace PACS Enterprise; Philips North America). Measurements on MRIs uploaded to PACS from outside the study institution performed using the same tool.

Labral width measurements were made at 3 standardized locations of the superior to anterior labrum using the clockface paradigm. ${ }^{10}$ For consistency, previously established anatomical landmarks were used. ${ }^{10}$ Measurements were made at the 11:30-o'clock position on coronal proton density (PD) sequence using the posterior border of the indirect head of the rectus femoris tendon as the landmark, at 3-o'clock position on axial oblique PD sequence where the iliopsoas tendon passes anterior to the labrum corresponding to the psoas $\mathrm{U}$, and a point halfway between the 2 aforementioned positions on sagittal fat suppressed PD representing 1:30-o'clock position (anterosuperior labrum) (Fig 1). The radial series was not present in all MRIs and, therefore, was not included for purposes of standardization.

Labral width in mm was measured from the acetabular rim to the free edge of the labrum along the outer surface of the labrum. The labral outer surface was chosen to correspond to the method of labral width measurement in arthroscopy. Radiologists were blinded to the results and outcomes of patients' surgery. This technique has been shown to have excellent interrater reliability in a prior study by the same study group. ${ }^{16}$

\section{Statistical Analysis}

An a-priori power analysis was performed before commencement to determine how many patients would be required to detect a statistical difference. A recent paper by Brinkman et al. ${ }^{17}$ evaluated labral widths amongst patients undergoing arthroscopic repair. Although this paper compared several groups, to ensure no type II error, groups with the smallest difference were used. This included the means of the bottom decile and bottom quartile ( $3.2 \mathrm{vs} 3.8 \mathrm{~mm}$ ) and their standard deviations (both $0.4 \mathrm{~mm}$ ). Setting alpha to 0.05 , and beta to $0.2(80 \%$ power), it was determined 7 patients would be required in each group. When the largest widths in their study were examined, the difference between groups was larger and the standard deviation was also larger. Using the means of the top quartile compared with the top decile (6.9 vs 8.4 $\mathrm{mm}$ ) and standard deviations (1.2 and 1.3), again with alpha at 0.05 , and beta at 0.2 , the power analysis determined 10 patients would be required in each group. 
- 147 patients without documented Beighton scores

- 64 patients without available MRIs

- 23 patients with evidence of dysplasia

- 2 patients with calcified labra

155 - 11 patients with MRI of insufficient quality

- 17 patients with high Beighton scores

142 - Matched cohort created from remaining 125 patients

Fig 2. Patient inclusion sequence. (MRI, magnetic resonance imaging.)

For comparison, patients were divided into groups of high Beighton score $(\geq 4)$ or low Beighton scores $(<4)$ based on stratification performed in previous studies that have investigated the impact of GJH on outcomes in hip arthroscopy. ${ }^{18}$ The most frequent cutoff for GJH was $\geq 4$. $^{6}$

Statistical analysis was performed using Mann-Whitney $U$, Fisher exact testing, and linear regression. Mann-Whitney $U$ analysis was performed instead of paired $t$ tests, as the labral width data was not found to be normally distributed each position in either cohort. Fisher exact analysis was used instead of $\chi^{2}$ testing as expected counts were less than 5 in at least 1 cell. Intraclass correlation coefficient testing was used to assess inter-rater reliability.

\section{Results}

A total of 242 patients underwent hip arthroscopy for FAI and had documented Beighton scores during the study period. A total of 389 primary hip arthroscopies were performed during the study period. Of the 242 patients, 178 patients had MRIs available for review. Of these, 23 had evidence of dysplasia and were excluded. Of the remaining 155 patients, 2 patients had calcified labra (agreed upon by both readers) and 11 patients had MRI of insufficient quality for analysis. Of the remaining 142 patients, 17 patients had Beighton scores $\geq 4$ (range 4-9, mean 5.47) A matched cohort of 17 controls with Beighton scores $<4$ (range 0-2, mean 1.50) was selected from the remaining 125 patients (Fig 2).

Thirty-four patients met inclusion criteria, including 17 cases and 17 controls. Both groups were composed exclusively of female patients. There was no significant difference between cases or controls in terms of age $(33.3 \pm 10.4$ vs $35.2 \pm 8.3$ years $P=.57)$ or BMI $(26.1$ \pm 9.3 vs $23.6 \pm 3.4, P=.36$ ). There was no significant difference in laterality between groups ( 8 right hips, 9 left for the high Beighton score group, 10 right and 7 left for controls, $P=.31$ ). There were significantly more patients with connective tissue disorders between groups (6 for the high Beighton score group vs 0 , $P=.011)$. Lateral center edge angle was $\geq 25^{\circ}$ in both groups, and there was no significant difference between cases and controls $(31.0 \pm 5.2$ vs $31.8 \pm 3.0, P=.58)$. Demographic information can be found in Table 1 . Inter-rater labral width reliability was excellent at all positions measured (Table 2).

The high Beighton score cohort had thinner labrae at all locations measured but one. This included significantly thinner labrae at the indirect rectus $(5.35$ $\mathrm{mm} \pm 1.2$ vs $7.1 \mathrm{~mm} \pm 1.1, P<.001)$ and anterosuperior position $(5.53 \mathrm{~mm} \pm 1.4 \mathrm{vs} 7.27 \mathrm{~mm} \pm 1.6$, $P=.003$ ). There was no statistical difference between the high Beighton score cohort and controls at the Psoas U position $(6.47 \mathrm{~mm} \pm 1.6 \mathrm{vs} 7.43 \mathrm{~mm} \pm 1.7, P=.112)$ (Table 3).

Linear regression analysis demonstrated Beighton score was significantly negatively associated with labrum width at the indirect rectus position $\left(\mathrm{R}^{2}=0.33\right.$, $P<.001)$ and the anterosuperior position $\left(\mathrm{R}^{2}=0.25\right.$,

Table 1. Demographic Data

\begin{tabular}{|c|c|c|c|}
\hline & \multicolumn{2}{|c|}{ Demographics } & \multirow[b]{2}{*}{$P$ Value } \\
\hline & High Beighton Score & Controls & \\
\hline Total number of patients & 17 & 17 & \\
\hline Age, y & $33.3 \pm 10.4$ & $35.2 \pm 8.3$ & .57 \\
\hline \multicolumn{4}{|l|}{ Sex } \\
\hline Female & 17 & 17 & \\
\hline Male & 0 & 0 & \\
\hline Laterality & & & .308 \\
\hline Body mass index & $26.1 \pm 9.3$ & $23.6 \pm 3.4$ & .36 \\
\hline Lateral center edge angle, $^{\circ}$ & $31 \pm 5.2$ & $32.8 \pm 3.0$ & .58 \\
\hline Patients with connective tissue disorders & 6 & 0 & .011 \\
\hline
\end{tabular}

NOTE. Significant $P$ values are bolded. 
Table 2. Reliability Analysis With ICC Assessment

\begin{tabular}{|c|c|c|c|c|}
\hline \multirow[b]{2}{*}{ MRI (Anatomic Location) } & \multirow[b]{2}{*}{ Width, mm Reader 1} & \multirow[b]{2}{*}{ Width, mm Reader 2} & \multicolumn{2}{|l|}{ ICC } \\
\hline & & & Radiologist Inter-Rater & $P$ Value \\
\hline Psoas-U (3 o'clock) & $6.9 \pm 1.7$ & $7.26 \pm 1.6$ & 0.92 & $<.001$ \\
\hline Indirect rectus (11:30 o'clock) & $6.2 \pm 1.4$ & $6.4 \pm 1.4$ & 0.91 & $<.001$ \\
\hline
\end{tabular}

NOTE. Significant $P$ values are bolded.

ICC, intraclass correlation coefficient; MRI, magnetic resonance imaging.

$P=.004)$. Beighton score was not significantly associated with labrum width at the Psoas U position $(P=.107)($ Table 4$)$.

\section{Discussion}

The most important finding from this study is that patients with GJH, defined as BTS $\geq 4$, were found to have significantly thinner labra than those without GJH (BTS <4). The clinical significance of these results relates to the ability to predict preoperatively patients who are likely to have thinner labra, which may have implications in terms of preoperative counseling, intraoperative labral management and anticipated postoperative outcomes.

Patients with greater Beighton scores are predisposed to hip microinstability, which is a poorly recognized clinical entity that can substantially contribute to pain and functional deficits in young, active patients. 8,19,20 The importance of the anterior hip capsule for conferring stability to the hip in patients with GJH is becoming increasingly evident. Repetitive stress on the anterior hip capsule can lead to increased tensile forces within the hip joint, predisposing patients to injury at the chondrolabral junction. ${ }^{19}$ As a result, patients with high Beighton scores are at increased risk for developing labral injuries. In the present study, an objective factor, labral width, was identified, which may contribute to why patients with GJH are more likely to incur hip labral injuries.

Saadat et al. ${ }^{18}$ found that among a cohort of 1381 patients with GJH undergoing hip arthroscopy, 261 patients had Beighton scores $\geq 4$, and $92.7 \%$ of these patients were female. Authors of the study found that in patients with BTS $\geq 4,90.8 \%$ underwent labral repair, as compared with $74.0 \%$ of patients with BTS of 0 and $75.5 \%$ of patients with BTS $<4$. This is consistent with the notion that patients with elevated BTS appear to be predisposed to labral injury compared with those with BTS in the normal range. In addition, Saadat et al. ${ }^{18}$ found that patients with GJH had smaller intraoperative labral size than those without GJH, which is also consistent with our MRI-based measurement of labral size. In this context, labral width can be reliably measured via MRI preoperatively to provide the surgeon with more information that can assist with planning in anticipation of intraoperative findings. This justifies the use of MRI-based measurement for labral width as was performed in this study, as opposed to an intraoperative measurement.

Of note, the rates of GJH in the literature appear to vary. Saadat et al. ${ }^{18}$ report a $20 \%$ rate of hyperlaxity among a cohort of 1381 patients who underwent hip arthroscopy for FAI, and Naal et al. ${ }^{6}$ report a $32.7 \%$ rate of hyperlaxity among a cohort of 55 patients with a diagnosis of FAI. Our study identified approximately a $12 \%$ rate of $\mathrm{GJH}$, which is lower than the other reports. This may be a function of discrepancies in Beighton scoring methodologies between the studies; however, selection bias cannot be excluded as a contributing factor.

In general, hip arthroscopists rely on intraoperative findings to dictate management of labral pathology. ${ }^{21}$ Factors that surgeons consider when deciding how to manage the labrum include the tear pattern, tear size, the stability of the labrum to probing, labral tissue quality, labral calcification, and thickness of the labrum. $^{21-23}$ Moreover, Domb et al. ${ }^{22}$ and Phillipon et al. ${ }^{23}$ both suggested that a hypoplastic labrum identified intraoperatively may be an indication for labral reconstruction, as opposed to a primary labral repair. Identification of a thin labrum preoperatively would greatly assist in planning before surgery. This

Table 3. Comparisons of Labral Widths

\begin{tabular}{lcrr}
\hline \multicolumn{1}{c}{ Labrum } & High Beighton & \multicolumn{1}{c}{ Controls } \\
\multicolumn{1}{c}{ Location } & Scores & $7.1 \pm 1.1$ & $P$ Value \\
\hline Indirect rectus (11:30 o'clock) & $5.35 \pm 1.2$ & $7.27 \pm 1.6$ & $\mathbf{0 0 1}$ \\
Anterosuperior position (1:30 o'clock) & $5.53 \pm 1.4$ & $7.43 \pm 1.7$ & $\mathbf{. 0 0 3}$ \\
Psoas-U (3:00 o'clock) & $6.47 \pm 1.6$ & $\mathbf{. 1 1 2}$ \\
\hline
\end{tabular}

NOTE. Values are shown in millimeters. Significant $P$ values are bolded. 
Table 4. Linear Regression Correlations for Labrum Locations

\begin{tabular}{lcc}
\hline \multicolumn{1}{c}{ Labrum } & & \\
Location & $\mathrm{R}^{2}$ & $P$ Value \\
\hline Indirect rectus (11:30 o'clock) & 0.33 & $<.001$ \\
Anterosuperior & 0.25 & $\mathbf{. 0 0 4}$ \\
Position (1:30) o'clock & & \\
Psoas-U (3:00 o'clock) & 0.054 & .12 \\
\hline
\end{tabular}

NOTE. Values are shown in millimeters. Significant $P$ values are bolded.

information would allow surgeons to better counsel patients on expected intraoperative findings and management decisions. It would also facilitate ensuring that all necessary equipment is available in the operating room if labral reconstruction is ultimately undertaken. This study adds to the body of evidence that underscores not only the value of identifying labral width preoperatively, but also that patients with GJH, which comprise a substantial proportion of hip arthroscopy patients, are predisposed to decreased labral width. As nearly $20 \%$ of patients undergoing hip arthroscopy have $\mathrm{GJH}^{18}{ }^{18}$ it behooves surgeons to consider the unique anatomical features of this population.

The outcomes of hip arthroscopy in patients with GJH have been previously investigated. Maldonado et al. ${ }^{24}$ found that patients with GJH who underwent hip arthroscopy for symptomatic FAI and labral tears experienced favorable outcomes with appropriate labral and capsular management at minimum 2-year followup when compared with a control cohort without GJH. Moreover, Pontiff et al. ${ }^{25}$ compared the outcomes of 35 women with GJH (defined as BTS $\geq 4$ ) and 131 women without GJH and found similar patientreported outcomes between the cohorts. However, the follow-up period in this study was only 6 months, thus limiting the generalizability of the results. Stone et al. ${ }^{26}$ similarly investigated the clinical outcomes among patients with and without GJH (defined as BTS $\geq 4$ ) with 2 years of follow-up and found similar improvement in both cohorts based on $\Delta$ value for mHHS $(P=.913)$, HOS-SSS $(P=.944)$, HOS-Activities of Daily Living $(P=.618)$, and VAS $(P=.512)$. Therefore, the existing literature appears to support that patients with GJH who undergo hip arthroscopy with appropriate capsular management and labral restoration perform comparably to those patients without GJH.

\section{Limitations}

Several factors should be considered when interpreting the results of this study. First, this study consisted of all female subjects, which may be interpreted as a limitation. This is consistent with other publications, which have found labral tears to be more common in women than men. ${ }^{27,28}$ In addition, GJH is also more prevalent in female than in male patients. Other investigations that have similarly compared outcomes of hip arthroscopy in patients with and without GJH have included primarily female patients only to be consistent between the experimental and control cohorts. As such, providers should be cautious when applying the results of this study to male patients. Also, as FAI was an inclusion criterion, there may have been some patients eliminated with normal bony morphology but hip problems related to microinstability. In addition, labral width was measured on MRI, and not intraoperatively, which is the gold standard. However, measuring labral width via MRI has been shown to be a validated technique, ${ }^{16}$ thus interpreting the conclusions of this study is reasonable. Also, not all of the 242 patients who underwent hip arthroscopy for FAI identified for analysis in this study had MRI scans available for review. As such, there were a large number of patients with incomplete data, including incomplete documentation of Beighton score. This is an important limitation, as it does introduce the possibility of selection bias. The fact that the study did not include a consecutive series of patients also introduces selection bias. The conclusions certainly would be strengthened if none of the study patients were excluded. The MRI review did not include an assessment of femoral torsion or acetabular version, which also limits the results. Since clinical follow-up was not included in this study, clinical relevance of these results can be extrapolated but not concluded definitively. It is important to note that this study includes a lower number of patients with elevated BTS relative to other studies. Although our results do reach statistical significance, larger studies that include more patients may be useful in order to further support the findings of this study. Finally, intraoperative data not collected or reported include the presence of a labral tear, location or type of labral tear (if present), or the intraoperative procedure performed (labral repair vs debridement vs reconstruction).

\section{Conclusions}

Patients with a Beighton test score $\geq 4$ were found to have significantly thinner labra than those with a Beighton test score of $<4$.

\section{References}

1. Ferguson SJ, Bryant JT, Ganz R, Ito K. An in vitro investigation of the acetabular labral seal in hip joint mechanics. J Biomech 2003;36:171-178.

2. Bharam S. Labral tears, extra-articular injuries, and hip arthroscopy in the athlete. Clin Sports Med 2006;25: 279-292, ix.

3. Myers SR, Eijer H, Ganz R. Anterior femoroacetabular impingement after periacetabular osteotomy. Clin Orthop Rel Res 1999:93-99.

4. Ferguson SJ, Bryant JT, Ganz R, Ito K. The acetabular labrum seal: A poroelastic finite element model. Clin Biomech 2000;1 5:463-468. 
5. Russek LN, Errico DM. Prevalence, injury rate and, symptom frequency in generalized joint laxity and joint hypermobility syndrome in a "healthy" college population. Clin Rheumatol 2016;35:1029-1039.

6. Naal FD, Hatzung G, Muller A, Impellizzeri F, Leunig M. Validation of a self-reported Beighton score to assess hypermobility in patients with femoroacetabular impingement. Int Orthop 2014;38:2245-2250.

7. Safran MR. Microinstability of the Hip-Gaining Acceptance. J Am Acad Orthop Surg 2019;27:12-22.

8. Shu B, Safran MR. Hip instability: Anatomic and clinical considerations of traumatic and atraumatic instability. Clin Sports Med 2011 ;30:349-367.

9. McCrum CL. Editorial Commentary: Predicting the size of the labrum: We have the technology, should we put it to use? Arthroscopy 2020;36:759-760.

10. Philippon MJ, Nepple JJ, Campbell KJ, et al. The hip fluid seal-Part I: The effect of an acetabular labral tear, repair, resection, and reconstruction on hip fluid pressurization. Knee Surg Sports Traumatol Arthrosc 2014;22:722-729.

11. Kaplan DJ, Samim M, Burke CJ, Baron SL, Meislin RJ, Youm T. Decreased hip labral width measured via preoperative MRI is associated with inferior outcomes for arthroscopic labral repair for femoroacetabular impingement. Arthroscopy 2021;37:98-107.

12. Beltran LS, Rosenberg ZS, Mayo JD, et al. Imaging evaluation of developmental hip dysplasia in the young adult. AJR Am J Roentgenol 2013;200:1077-1088.

13. Leunig M, Podeszwa D, Beck M, Werlen S, Ganz R. Magnetic resonance arthrography of labral disorders in hips with dysplasia and impingement. Clin Orthop Relat Res 2004:74-80.

14. Boyle KL, Witt P, Riegger-Krugh C. Intrarater and interrater reliability of the beighton and Horan Joint Mobility Index. J Athl Train 2003;38:281-285.

15. Wolf JM, Cameron KL, Owens BD. Impact of joint laxity and hypermobility on the musculoskeletal system. J Am Acad Orthop Surg 2011;19:463-471.

16. Kaplan DJ, Samim M, Burke CJ, Meislin RJ, Youm T. Validity of magnetic resonance imaging measurement of hip labral width compared with intraoperative assessment. Arthroscopy 2020;36:751-758.

17. Brinkman JC, Domb BG, Krych AJ, et al. Is labral size predictive of failure with repair in hip arthroscopy? Arthroscopy 2020;36:2147-2157.
18. Saadat AA, Lall AC, Battaglia MR, Mohr MR, Maldonado DR, Domb BG. Prevalence of generalized ligamentous laxity in patients undergoing hip arthroscopy: A prospective study of patients' clinical presentation, physical examination, intraoperative findings, and surgical procedures. Am J Sports Med 2019;47:885-893.

19. Shindle MK, Ranawat AS, Kelly BT. Diagnosis and management of traumatic and atraumatic hip instability in the athletic patient. Clin Sports Med 2006;25:309-326, ix-x.

20. Blakey CM, Field MH, Singh PJ, Tayar R, Field RE. Secondary capsular laxity of the hip. Hip Int 2010;20: 497-504.

21. Herickhoff PK, Safran MR. Surgical decision making for acetabular labral tears: An international perspective. Orthop J Sports Med 2018;6:2325967118797324.

22. Domb BG, Hartigan DE, Perets I. Decision making for labral treatment in the hip: repair versus debridement versus reconstruction. J Am Acad Orthop Surg 2017;25: e53-e62.

23. Philippon MJ, Briggs KK, Fagrelius T, Patterson D. Labral refixation: current techniques and indications. HSS J 2012;8:240-244.

24. Maldonado DR, Chen JW, Yelton MJ, et al. Achieving successful outcomes of hip arthroscopy in the setting of generalized ligamentous laxity with labral preservation and appropriate capsular management: A propensity matched controlled study. Am J Sports Med 2020;48: 1625-1635.

25. Pontiff M, Ithurburn MP, Ellis T, Cenkus K, Stasi SD. Pre- and post-operative self-reported function and quality of life in women with and without generalized joint laxity undergoing hip arthroscopy for femoroacetabular impingement. Int $J$ Sports Phys Ther 2016;11:378-387.

26. Stone AV, Mehta N, Beck EC. Comparable patient-reported outcomes in females with or without joint hypermobility after hip arthroscopy and capsular plication for femoroacetabular impingement syndrome. J Hip Preserv Surg 2019;6:33-40.

27. Burnett RS, Della Rocca GJ, Prather H, Curry M, Maloney WJ, Clohisy JC. Clinical presentation of patients with tears of the acetabular labrum. J Bone Joint Surg Am 2006;88:1448-1457.

28. Dorrell JH, Catterall A. The torn acetabular labrum. J Bone Joint Surg Br 1986;68:400-403. 